\title{
Adaptação e eficiência de um índice para análise da integridade biótica em Floresta Ombrófila Densa
}

\author{
Adaptation and efficiency of an index for bioty integrity analysis in Tropical \\ Rain Forest
}

Pietro de Oliveira Scarascia', Roberta Averna Valente II, Eliana Cardoso-Leite ${ }^{\mathrm{III}}$

\section{Resumo}

Diante dos diversos métodos de mensuração da integridade biótica de determinada área de floresta evidencia-se a Avaliação Ecológica Rápida (AER). Com base na AER foi desenvolvido um índice para avaliar a condição da vegetação, denominado Índice de Integridade Biótica (IIB). A aferição e precisão deste índice retratam o quão íntegro um dado ecossistema florestal se encontra no momento da análise. O alicerce para a eficiência na aplicação do IIB está na escolha correta dos indicadores de integridade biótica, ou seja, nas variáveis a serem mensuradas. O presente estudo partiu de um índice anteriormente proposto para Floresta Estacional Semidecidual (FES), e teve como objetivo adaptar o IIB para uso em área de Floresta Ombrófila Densa no Estado de São Paulo, bem como testar sua eficiência no diagnóstico da integridade de fragmentos de Floresta Ombrófila Densa (FOD) inseridos em matriz rural. Dos onze indicadores presentes no método original, alguns foram mantidos como no original ou adaptados, alguns retirados e alguns novos foram criados. O IIB adaptado foi aplicado numa área de FOD de 70 hectares. Para isso, foram utilizadas 20 parcelas de $10 \times 10 \mathrm{~m}$, dentro da área amostral. Os resultados mostraram uma variação do IIB entre 28 e 47, ou seja, 2 parcelas registraram integridade baixa, 11 integridade média e 7 integridade boa. A área como um todo apresentou integridade regular, o que era esperado devido ao seu tamanho, ao entorno agrícola e ao histórico de perturbações. O IIB mostrou-se adaptado e eficiente para análise da integridade biótica da FOD, pois conseguiu mostrar a diferença de integridade entre diferentes trechos de Floresta Ombrófila Densa. Os indicadores menos eficientes foram: "cobertura de serapilheira" e "espécies exóticas lenhosas". Os mais eficientes foram: "Euterpe edulis", "epífitas", "clareiras" e "cipós/lianas".

Palavras-chave: Conservação; Gestão de áreas protegidas; Planejamento territorial

\begin{abstract}
Among the different ways of measuring the biotic integrity of a particular forest area, the Rapid Ecological Assessment (REA) stands out. Based on REA, an index was developed to evaluate the quality of the vegetation called the Biotic Integrity Index (BII). The measurement and accuracy of this index show how integral is the forest ecosystem at moment of analysis. The basis for efficiency in the application of BII is the correct choice of biotic integrity indicators or the variables to be measured. The present study was based from an index previously proposed for a Seasonal Semideciduous Forest (SSF), and aimed to adapt the BII for use in an area of Dense Ombrophilous Forest (DOF) in the State of São Paulo as well as to test its efficiency in diagnosing the integrity of fragments of a Tropical Rain Forest (TRF) inserted in a rural matrix. From the eleven indicators present in the original method, some were kept as original or adapted, some removed and some new ones were created. The adapted BII was applied in a TRF area of 70 hectares. To do so, 20 plots of $10 \times 10 \mathrm{~m}$ were used, within the sampling area. The results showed a variation of IIB from 28 to 47, and 2 plots recorded low integrity, 11 medium integrity and 7 good integrity. The area showed regular integrity as a whole, which was expected due to its size, agricultural surrounding and history of disturbances. The BII showed to be adapted and efficient to analyze the biotic integrity of the DOF, as it was able to show the difference of integrity between different patches of TRF. The least efficient indicators were: "litter cover" and "exotic woody species", and the most efficient were: "Euterpe edulis", "epiphytes", "gaps" and "vines".
\end{abstract}

Keywords: Conservation; Management of protected areas; Territorial planning

\footnotetext{
Médico Veterinário, MSc., Gestor do Parque Estadual Carlos Botelho, Fundação Florestal, Secretaria de Infraestrutura e Meio Ambiente do Estado de São Paulo, Rodovia SP139 km78, CEP18230-000, São Miguel Arcanjo (SP), Brasil. pietroos@fflorestal.sp.gov.br (ORCID: 0000-0002-1056-5641) Engenheira Florestal, Dra., Professora do Departamento Ciências Ambientais e do Programa de Pós-Graduação em Planejamento e Uso dos Recursos Renováveis do Centro de Ciências e Tecnologia para Sustentabilidade da Universidade Federal de São Carlos - campus Sorocaba, Rodovia João Leme dos Santos (SP 264) - Km 110, bairro Itinga, CEP 18052780, Sorocaba (SP), Brasil. roavalen@ufscar.br (ORCID: 0000-0001-7273-7042)

III Bióloga, Dra., Professora do Departamento de Ciências Ambientais e do Programa de Pós-Graduação em Sustentabilidade na Gestão Ambiental do Centro de Ciências e Tecnologia para Sustentabilidade da Universidade Federal de São Carlos - campus Sorocaba, Rodovia João Leme dos Santos (SP 264) - Km 110, bairro Itinga, CEP 18052780, Sorocaba (SP), Brasil. eliana.leite@ufscar.br (ORCID: 0000-0003-3152-2576)
} 


\section{Introdução}

As pressões de destruição da biodiversidade apresentam grande patamar dentro da história da humanidade. Frente a essa perspectiva, é urgente a necessidade de execução de ações que objetivem a preservação e a conservação dos ecossistemas florestais, principalmente do bioma Mata Atlântica, onde se observa um cenário ainda mais severo quando comparado a outros ecossistemas terrestres (MITTERMEIER et al., 1998; PAVIOLO et al., 2016).A criação e manutenção de Unidades de Conservação têm sido a principal estratégia adotada por muitos países, na tentativa de conservar os ecossistemas e diversidade biológica a eles associada. No entanto, para criar e manejar com eficiência essas áreas protegidas, é necessário conhecer o seu status de conservação ou a sua integridade biótica (HOCKINGS; STOLTON; DUDLEY, 2000).

Assim, a análise de sua vegetação é uma importante maneira de avaliar o status de conservação de um ecossistema terrestre (SICHE et al., 2007; CASTELLO; COELHO; CARDOSO-LEITE, 2017), pois os componentes da comunidade vegetal apresentam respostas às variações ambientais por se tratarem de organismos pouco tolerantes às alterações causadas pela degradação (BOSCOLO et al., 2017). Dessa forma, diversos autores (SICHE et al., 2007; CASTELLO; COELHO; CARDOSO-LEITE, 2017; GRACIANO-SILVA; MELLO; CARDOSOLEITE, 2018) utilizam componentes da vegetação para planejar e estimar indicadores de estado de conservação em áreas protegidas.

Diante dos métodos de mensuração da integridade biótica de determinada área, destacase a Avaliação Ecológica Rápida (AER) (SAYRE et al., 2000). A AER é uma metodologia muito utilizada no diagnóstico da biodiversidade para planejamento conservacionista de um território (SOBREVILLA; BATH, 1992; SAYRE et al., 2000; SUTULA et al., 2006; ALLEN, 2009; STEIN; FETSCHER; CLARK 2009). A partir da AER, Medeiros e Torezan (2013) desenvolveram um índice de análise da vegetação para Floresta Estacional Semidecidual, denominado Índice de Integridade Biótica (IIB). A mensuração e acurácia deste índice retratam o quão íntegro um dado ecossistema está no momento da análise (GREGORINI, 2015; GRACIANO-SILVA, 2016; CASTELLO; COELHO; CARDOSO-LEITE, 2017).

O alicerce para a eficiência na aplicação do IIB está na escolha correta dos indicadores de integridade biótica, ou seja, das variáveis a serem mensuradas. Esses indicadores devem representar e fornecer, com confiança, informações sobre a condição do ecossistema e de sua integridade biótica. É necessário que sejam selecionados indicativos de singularidade representativos para sua composição, a fim de serem traduzidos quantitativamente e possibilitar comparações (GRACIANO-SILVA; MELLO; CARDOSO-LEITE, 2018).

Os indicadores subsidiam melhoria na eficiência de gestão de áreas protegidas, uma vez que, dentro do resultado, pode-se classificar a integridade biótica da área analisada e entender a sua necessidade de manejo: se direcionado à recuperação ou à conservação. Proporcionando um diagnóstico da área, o IIB é uma ferramenta promissora para avaliar se os objetivos de conservação foram alcançados ou não, possibilitando então o seu monitoramento (LE SAOUT et al., 2013; CASTELLO; COELHO; CARDOSO-LEITE, 2017).

O presente estudo partiu de um índice de integridade biótica proposto por Medeiros e Torezan (2013) para Floresta Estacional, e teve como objetivo adaptá-lo para uso em área de Floresta Ombrófila Densa no Estado de São Paulo, bem como testar a eficiência do índice adaptado na análise da integridade de uma área Floresta Ombrófila inserida em matriz rural, analisando os mesmos comparativamente com Floresta Ombrófila Densa bem preservada e gerida como Parque Estadual (BRASIL, 2000).

Partiu-se do pressuposto que a área do Parque Estadual de Carlos Botelho, utilizada como referência, deveria apresentar maior integridade biótica que a área da Reserva Particular, pois a área do Parque Estadual é maior, com entorno florestal e a área da Reserva Particular é menor, com entorno agrícola. 


\section{Materiais e métodos}

\section{Caracterização da área de estudo}

O estudo foi conduzido na Reserva Particular do Patrimônio Natural Trápaga, Unidade de Conservação (UC), privativa do grupo de Uso Sustentável, criada pela Resolução SMA n ${ }^{\circ} 10$, de 30 de janeiro de 2018. Situada no interior da Fazenda Lageado, em São Miguel Arcanjo, Estado de São Paulo, entre as coordenadas UTM 23 J 1967077336140 e 23 J 197452 7337450, a RPPN Trápaga possui uma área de 70 hectares (Figura 1). O Parque Estadual Carlos Botelho (PECB), Unidade de Conservação do grupo de Proteção Integral, com 38.705 hectares, localizado ao lado da RPPN de Trápaga, foi utilizado como área de referência no presente estudo.

Figura 1 - Localização da RPPN Trápaga: no Estado de São Paulo, no Mosaico do Paranapiacaba, na Zona de Amortecimento do PECB, na Fazenda Lageado

Figure 1 - Location of the RPPN Trápaga: in the state of São Paulo, in the Mosaic of Paranapiacaba, in the CBSP Buffer Zone, at Lageado Farm

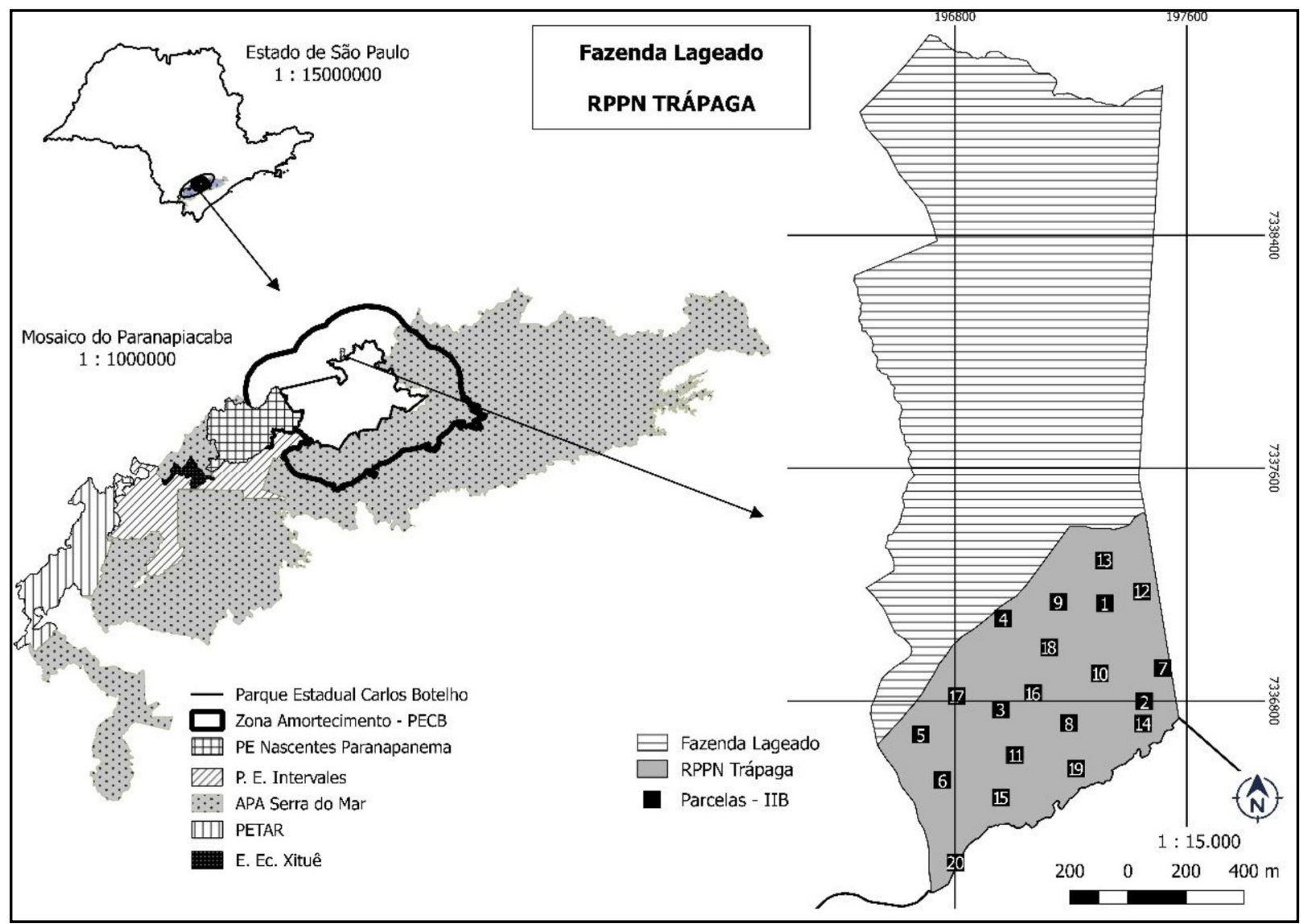

Fonte: Autores (2018) 


\section{Método utilizado}

Para avaliar a qualidade do ecossistema florestal dentro da área da RPPN de Trápaga foi utilizado um índice de integridade biótica. Esse índice, originalmente proposto por Medeiros e Torezan (2013), avalia a integridade de ecossistemas florestais por meio dos indicadores: 1-cobertura de serapilheira, 2-árvores mortas em pé, 3-cobertura de gramíneas exóticas, 4-outras espécies exóticas, 5-lianas, 6-eco-unidades, 7-epífitas vasculares (exceto orquídeas), 8-orquídeas, 9- figueiras (Ficus ssp), 10-palmeiras (Euterpe edulis) e 11-peroba rosa (Aspidosperma polyneuron). Partindo-se do IIB original (MEDEIROS; TOREZAN, 2013), que foi proposto para FES (Floresta Estacional Semidecidual), foram feitas adaptações para ser utilizado em FOD (Floresta Ombrófila Densa). Cada indicador foi revisto, no sentido de ser mantido, alterado ou retirado, e novos indicadores foram propostos. Os principais estudos utilizados para embasar essa adaptação foram os realizados pelo Plano de Manejo do Parque Estadual Carlos Botelho (INSTITUTO FLORESTAL, 2008) que apresenta aspectos ecológicos dos ecossistemas presentes na região, e o estudo de Castello, Coelho e Cardoso-Leite (2017) que analisou indicadores de conservação para FOD, no Estado de São Paulo, em região próxima à estudada.

Assim, os indicadores "cobertura de serapilheira", "árvores mortas em pé" e "cobertura de gramíneas exóticas” foram mantidas como no método original, apenas adequando os descritores (graus de variação, que recebem nota de 1 a 5), como apresentado na Tabela 1. Esses indicadores foram mantidos, pois a serapilheira representa a função de ciclagem de nutrientes nos ecossistemas (TURCHETTO; FORTES, 2014). A mortalidade de árvores indica a qualidade ambiental, podendo auxiliar no reconhecimento de perturbações existentes (FRANKLIN; SHUGART; HARMON, 1987; GASPER et al., 2013), e os impactos causados pela presença de espécies invasoras são evidentes (PIVELLO; FREITAS, 2008), sendo que as gramíneas africanas representam uma das espécies exóticas de maior capacidade invasora, como a Brachiaria spp. (BIZ et al., 2012).

O indicador "outras exóticas" foi transformado em "Espécies exóticas lenhosas". A invasão por espécies exóticas lenhosas é considerada a segunda maior causa de perda de biodiversidade no mundo. Foram consideradas as seguintes espécies exóticas: Pinnus elliottii, que é uma espécie exótica com alto potencial de invasão, além de espécies de limão (Citrus spp.), caqui (Diospyros kaki L.), ameixa (Eriobotrya japonica (Thunb.) Lindl.), açaí (Euterpe oleracea Mart.), eucalipto (Eucalyptus grandis W. Hill) e araucária (Araucaria angustifolia Kuntze), pois essas são as espécies exóticas mais frequentes na região (INSTITUTO FLORESTAL, 2008).

O indicador "lianas" foi mantido, pois a presença de cipós lenhosos é associada com melhoria na qualidade florestal (FONSECA; OLIVEIRA, 1998, CASTELLO; COELHO; CARDOSO-LEITE, 2017). Foi utilizado o trabalho de Castello, Coelho e Cardoso-Leite (2017) para definir as classes de diâmetro das lianas lenhosas, ou seja, para estabelecer o valor dentro das classes (1 a 5) para este indicador.

No método original (MEDEIROS; TOREZAN, 2013) o indicador "eco-unidades" foi descrito com uma mistura de critérios envolvendo desde o "tamanho das clareiras existentes" até "a altura das árvores do dossel superior". Dessa forma, este foi substituído por "clareiras" de modo a deixar a avaliação mais objetiva. A presença de clareiras ocasionada por queda de árvores é um dispositivo de manutenção da biodiversidade em florestas tropicais, uma vez que fornecem nichos distintos de colonização e a coexistência de espécies com diferentes histórias de vida em um mesmo local (GANDOLFI; JOLY; LEITÃO FILHO, 2009).

Os indicadores "epífitas vasculares" e "orquídeas" foram unidos, e este indicador foi denominado "epífitas". As epífitas são apontadas como indicadoras do estado de conservação de ecossistemas, devido a sua dependência da umidade atmosférica, de sombreamento e do substrato proporcionados por indivíduos arbóreos (MANIA, 2013). Assim, foram consideradas as bromélias (Bromeliaceae), orquídeas (Orchidaceae) e espécies de Cactaceae (Rhipsalis spp). 
O indicador "palmeiras (Euterpe edulis)" foi mantido, sendo considerada a presença de indivíduos desta espécie dossel. Essa é uma espécie arbórea listada como "Criticamente em Perigo" de extinção (sensu INTERNATION UNION FOR CONSERVATION OF NATUREZA, 2019), devido à pressão que sofre pela extração ilegal de seu palmito. Sua grande dispersão de sementes somada à presença de avifauna, e condições específicas de ambiente para seu desenvolvimento (umidade, sombra e calor), tornam essa espécie um item chave na manutenção da biodiversidade da Mata Atlântica (INSTITUTO FLORESTAL, 2008). Castello, Coelho e Cardoso-Leite (2017) registraram essa espécie como indicadora de florestas bem conservadas em áreas de Mata Atlântica, no Vale do Ribeira.

No método original os indicadores "Ficus spp", e "Aspidosperma spp" se referem a espécies "indicadoras", selecionadas pelos autores para a região em análise. Sendo assim, optouse em uni-los em um único indicador denominado "espécies tardias do dossel". Para a seleção de espécies tardias do dossel, elas foram consideradas de grande importância ecológica para a manutenção dos ecossistemas da região da área de estudo, selecionadas a partir de estudos prévios (INSTITUTO FLORESTAL, 2008; CASTELLO; COELHO; CARDOSO-LEITE, 2017). Foram consideradas as seguintes famílias/espécies: Lauraceae (Ocotea moseniiMez e Cryptocarya aschersoniana Mez); Lecythidaceae (Cariniana estrellensis RaddiKuntze) e Rubiaceae (Bathysa australis (A.St.-Hil. K.Schum)).

Foram criados dois novos indicadores. O indicador "espécies tardias no sub-bosque" e o indicador "fetos arborescentes" (Tabela 1).

Considerou-se a presença ou ausência de fetos arborescentes, ou xaxim, (Dicksonia spp. L’Hér), para análise deste indicador. Essa é uma espécie listada como "Em Perigo" de extinção (sensu, INTERNATION UNION FOR CONSERVATION OF NATUREZA 2019), devido a extração desenfreada das fibras oriundas de seu pseudocaule. Essa espécie possui crescimento lento, ocorre preferencialmente em locais sombreados, dessa forma, a sua ocorrência está associada à boa qualidade florestal (CASTELLO; COELHO; CARDOSO-LEITE, 2017).

Outro indicador incluído foi "espécies tardias de sub-bosque", pois estas vivem e completam seu ciclo de vida em ambiente sombreado, por isso podem ser consideradas indicadoras de floresta bem conservada (KOZERA; RODRIGUES; DITTRICH, 2008). Para analisar esse indicador foi considerada a presença de espécies das famílias Myrtaceae, Rubiaceae, Meliaceae (Trichilia spp.) e Arecaceae (Euterpe edulis) como indicadores de integridade (GRACIANO-SILVA; MELLO; CARDOSO-LEITE. 2018), e a presença de espécies de Piperaceae como indicador de degradação (CASTELLO; COELHO; CARDOSO-LEITE, 2017). Essas espécies/famílias foram selecionadas a partir de estudos realizados previamente na região (INSTITUTO FLORESTAL, 2007).

As variáveis ambientais utilizadas para mensurar a integridade biótica da área de estudo estão apresentadas na Tabela 1, e descritas a seguir. Essas variáveis foram coletadas entre setembro e dezembro de 2017, e analisadas conforme a pontuação descrita na Tabela 2.

Cada variável coletada recebeu uma nota (Tabela 1) que variou entre 1 (nota mínima) e 5 (nota máxima). Uma vez somadas as pontuações de cada indicador, o resultado foi analisado fornecendo o IIB de cada parcela (Tabela 2).

Para a coleta de dados, foram amostradas 20 parcelas, de 10 por 10 metros, ao longo dos 70 hectares da RPPN Trápaga, distribuídas de forma aleatória dentro da área de estudo. Foram também amostradas quatro parcelas na área de referência (Parque Estadual de Carlos Botelho) apenas para servir como "padrão" de comparação. Essas quatro parcelas foram distribuídas na área mais conservada do PECB (Zona Intangível) de modo a se obter a referência de área bem conservada para a região. No método original os autores (MEDEIROS; TOREZAN, 2013) utilizaram três parcelas para cada área amostrada, sendo assim, as quatro parcelas da área de referência podem ser consideradas suficientes para amostrar a sua condição. 
Tabela 1 - Indicadores utilizados para a análise do Índice de Integridade Biótica, seus atributos e pontuação para cada variável

Table 1 - Indicators used for the analysis of the Biotic Integrity Index, its attributes and punctuation for each variable

\begin{tabular}{|c|c|c|c|c|c|}
\hline \multirow[t]{2}{*}{ Variável } & \multicolumn{5}{|c|}{ Escala de integridade 1 (muito baixa) a 5 (muito alta) } \\
\hline & 1 & 2 & 3 & 4 & 5 \\
\hline $\begin{array}{l}\text { A-Cobertura de } \\
\text { serapilheira }\end{array}$ & Solo exposto & $1-25 \%$ & $26-50 \%$ & $51-75 \%$ & $76-100 \%$ \\
\hline $\begin{array}{l}\text { B-Árvores mortas } \\
\text { em pé }\end{array}$ & $>3$ & 3 & 2 & 1 & Ausente \\
\hline $\begin{array}{l}\text { C-Cobertura de } \\
\text { gramíneas exóticas }\end{array}$ & $\geq 50 \%$ & $25-50 \%$ & $11-25 \%$ & $1-10 \%$ & Ausente \\
\hline $\begin{array}{l}\text { D-Espécies exóticas } \\
\text { lenhosas }^{2}\end{array}$ & $>3$ & 3 & 2 & 1 & Ausente \\
\hline E-Cipós e lianas ${ }^{1}$ & 3 ou,+ finos & 2 finos & $\begin{array}{l}1 \text { fino ou } \\
\text { ausentes }\end{array}$ & $\begin{array}{c}\text { Finas + grossas } \\
\text { (diâmetro } \geq 4 \mathrm{~cm} \text { ) finas }\end{array}$ & $\begin{array}{c}\text { Apenas grossas } \\
\text { (diâmetro } \geq 4 \mathrm{~cm} \text { ) }\end{array}$ \\
\hline F-Clareiras & $\geq 50 \%$ & $25-50 \%$ & $11-24 \%$ & $1-10 \%$ & Ausente \\
\hline G-Epífitas ${ }^{1}$ & Ausente & $1-5$ & $6-10$ & $11-15$ & $>15$ \\
\hline $\begin{array}{l}\mathrm{H} \text {-Fetos } \\
\text { arborescentes }^{1}\end{array}$ & Ausente & 1 & 2 & 3 & $\geq 4$ \\
\hline I-Euterpe edulis ${ }^{1}$ & Ausente & $1-3$ & $4-6$ & $7-9$ & $\geq 10$ \\
\hline $\begin{array}{l}\text { J-Espécies tardias } \\
\text { no dossel }^{2}, 3\end{array}$ & Ausente & 1 & 2 & 3 & 4 \\
\hline $\begin{array}{l}\text { K-Espécies tardias } \\
\text { no sub-bosque }{ }^{1},{ }^{4}\end{array}$ & $\begin{array}{c}\text { Ausente + } \\
\text { Presença de } \\
\text { Piperaceae }\end{array}$ & $\begin{array}{c}1-2+ \\
\text { Presença de } \\
\text { Piperaceae }\end{array}$ & $\begin{array}{c}3-5+ \\
\text { Presença de } \\
\text { Piperaceae }\end{array}$ & $\begin{array}{c}6-9+\text { Ausência } \\
\text { Piperaceae }\end{array}$ & $\begin{array}{c}\geq 10+\text { Ausência de } \\
\text { Piperaceae }\end{array}$ \\
\hline
\end{tabular}

Fonte: Autores (2019)

Em que: 1 =Número de indivíduos, 2 = Número de espécies, 3 = Lauraceae (Ocotea moseniiMez, Cryptocary aschersoniana Mez), Lecythidaceae (Cariniana estrellensis (Raddi) Kuntze), Rubiaceae (Bathysa australis (A. St.-Hil.) K. Schum); 4 = Myrtaceae, Rubiaceae, Meliaceae (Trichilia) e Arecaceae (Euterpe edulis).

Tabela 2 - Relação entre a pontuação dos valores de IIB mensurados a campo e suas respectivas classes

Table 2 - Relationship between the score of the IIB values measured in the field and their respective classes

\begin{tabular}{lccc}
\hline & IIB - Valores & & IIB - Classes \\
\hline $\mathbf{5 0}-\mathbf{5 5}$ & 5 & Excelente \\
$\mathbf{4 0 - 4 9}$ & 4 & Bom \\
$\mathbf{3 0}-\mathbf{3 9}$ & 3 & Regular \\
$\mathbf{2 0}-\mathbf{2 9}$ & 2 & Baixo \\
$\mathbf{1 1}-\mathbf{1 9}$ & 1 & Muito baixo \\
\hline
\end{tabular}

Fonte: Medeiros e Torezan (2013) 
Tabela 3: Variáveis amostradas, suas pontuações por parcelas e o Índice de Integridade Biótica de cada parcela mensurada na RPPN Trápaga e área controle (PECB)

Table 3: Sampled variables, their scores by plots and the Biotic Integrity Index of each plot measured in the RPPN Trápaga and control area (CBSP)

\begin{tabular}{|c|c|c|c|c|c|c|c|c|c|c|c|c|c|}
\hline \multirow[b]{2}{*}{ Parcelas } & \multicolumn{11}{|c|}{ Variáveis } & \multirow[b]{2}{*}{ IIB - Valor } & \multirow[b]{2}{*}{ IIB - Classe } \\
\hline & A & B & C & D & E & $\mathbf{F}$ & G & $\mathbf{H}$ & I & $\mathbf{J}$ & $\mathbf{K}$ & & \\
\hline 1 & 5 & 2 & 4 & 5 & 1 & 1 & 5 & 4 & 1 & 1 & 5 & 34 & Regular \\
\hline 2 & 5 & 5 & 5 & 5 & 4 & 3 & 2 & 1 & 1 & 2 & 5 & 38 & Regular \\
\hline 3 & 5 & 5 & 5 & 5 & 1 & 2 & 1 & 2 & 1 & 1 & 5 & 33 & Regular \\
\hline 4 & 5 & 2 & 5 & 5 & 1 & 2 & 1 & 3 & 1 & 2 & 5 & 32 & Regular \\
\hline 5 & 5 & 3 & 5 & 5 & 1 & 2 & 1 & 5 & 1 & 3 & 4 & 35 & Regular \\
\hline 6 & 5 & 3 & 5 & 5 & 1 & 2 & 1 & 3 & 1 & 2 & 5 & 33 & Regular \\
\hline 7 & 5 & 3 & 5 & 5 & 4 & 4 & 3 & 3 & 5 & 1 & 5 & 43 & Bom \\
\hline 8 & 5 & 5 & 5 & 5 & 2 & 3 & 2 & 4 & 5 & 1 & 4 & 41 & Bom \\
\hline 9 & 5 & 2 & 5 & 5 & 1 & 2 & 2 & 3 & 1 & 1 & 4 & 31 & Regular \\
\hline 10 & 5 & 2 & 5 & 5 & 1 & 1 & 2 & 4 & 1 & 1 & 5 & 32 & Regular \\
\hline 11 & 5 & 1 & 3 & 5 & 1 & 1 & 1 & 5 & 1 & 1 & 4 & 28 & Baixo \\
\hline 12 & 4 & 3 & 4 & 5 & 1 & 1 & 1 & 3 & 1 & 1 & 5 & 29 & Baixo \\
\hline 13 & 5 & 5 & 5 & 5 & 4 & 4 & 2 & 1 & 1 & 3 & 3 & 38 & Regular \\
\hline 14 & 5 & 3 & 5 & 5 & 3 & 4 & 2 & 5 & 2 & 1 & 4 & 39 & Regular \\
\hline 15 & 5 & 4 & 5 & 5 & 5 & 4 & 5 & 2 & 5 & 2 & 5 & 47 & Bom \\
\hline 16 & 5 & 5 & 5 & 5 & 4 & 4 & 2 & 5 & 1 & 3 & 3 & 42 & Bom \\
\hline 17 & 5 & 3 & 5 & 5 & 5 & 4 & 3 & 4 & 1 & 2 & 4 & 41 & Bom \\
\hline 18 & 5 & 3 & 5 & 5 & 1 & 2 & 4 & 1 & 1 & 2 & 4 & 33 & Regular \\
\hline 19 & 5 & 3 & 5 & 5 & 4 & 4 & 4 & 5 & 3 & 2 & 3 & 43 & Bom \\
\hline 20 & 5 & 5 & 5 & 5 & 5 & 4 & 5 & 5 & 2 & 1 & 5 & 47 & Bom \\
\hline PECB & 5 & 5 & 5 & 5 & 5 & 4 & 5 & 1 & 5 & 2 & 5 & 47 & Bom \\
\hline PECB & 5 & 5 & 5 & 5 & 5 & 5 & 5 & 2 & 5 & 2 & 5 & 49 & Bom \\
\hline PECB & 5 & 4 & 5 & 5 & 5 & 5 & 5 & 5 & 5 & 2 & 5 & 51 & Excelente \\
\hline PECB & 5 & 4 & 5 & 5 & 5 & 5 & 5 & 4 & 5 & 2 & 5 & 50 & Excelente \\
\hline
\end{tabular}

Fonte: Autores (2019)

Em que: $\mathrm{A}$ = Cobertura de serapilheira; $\mathrm{B}$ = Árvores mortas em pé; $\mathrm{C}=$ Gramíneas exóticas; $\mathrm{D}=$ Espécies Exóticas lenhosas; $\mathrm{E}=$ Cipós e lianas; $\mathrm{F}=$ Clareiras; $\mathrm{G}=$ Epífitas; $\mathrm{H}$ = Fetos arborescentes; $\mathrm{I}=$ Euterpe edulis; $\mathrm{J}$ = Espécie tardia de dossel; $\mathrm{K}$ = Espécie tardia de sub-bosque. 
Figura 2 - Atributos e valores de IIB relacionados a cada variável mensurada na RPPN Trápaga. Os eixos Y mostram os dados mensurados a campo (IIB - valor), os valores de $\mathrm{X}$ representam o resultado da escala de integridade de cada parcela amostrada (IIB - classes de integridade)

Figure 2 - IIB attributes and values related to each variable measured in the Trápaga RPPN. The $\mathrm{Y}$ axis shows the data measured in the field (IIB - value), the values of $\mathrm{X}$ represent the result of the integrity scale of each sample plot (IIB - integrity classes)

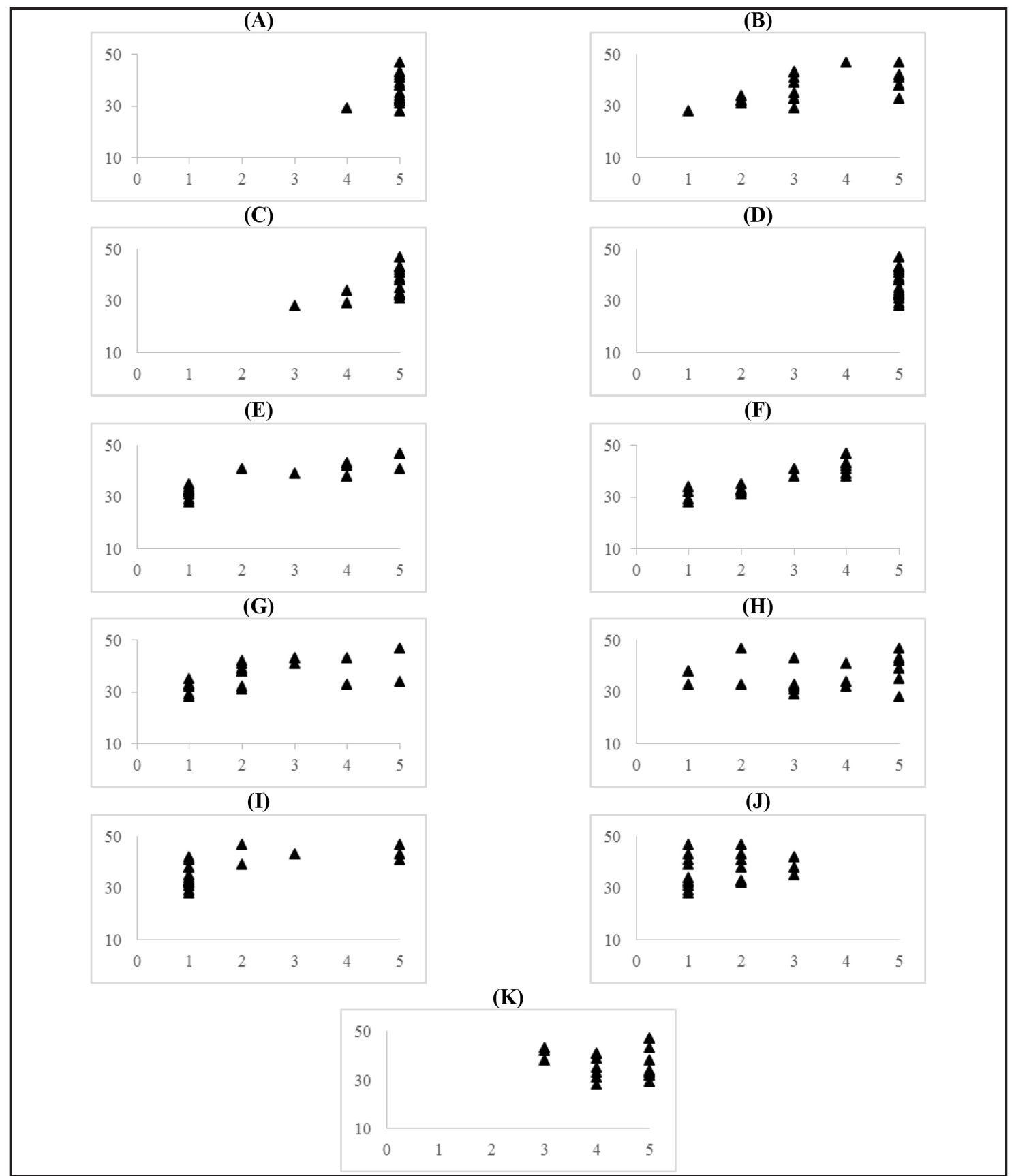

Fonte: Autores (2019)

Em que: $\mathrm{A}$ = Cobertura de serapilheira; $\mathrm{B}=$ Árvores mortas em pé; $\mathrm{C}=$ Gramíneas exóticas; $\mathrm{D}=$ Espécies Exóticas lenhosas; $\mathrm{E}=$ Cipós e lianas; $\mathrm{F}=$ Clareiras; $\mathrm{G}=$ Epífitas; $\mathrm{H}=$ Fetos arborescentes; $\mathrm{I}=$ Euterpe edulis; $\mathrm{J}=$ Espécie tardia de dossel; $\mathrm{K}$ = Espécie tardia de sub-bosque. 
Pode-se observar (Figura $2 \mathrm{~A}, \mathrm{D})$ que os indicadores A (cobertura de serapilheira) e D (espécies exóticas lenhosas) não foram eficientes, pois a serapilheira estava presente na grande maioria das parcelas e as espécies exóticas ausentes. O primeiro (serapilheira) também não foi considerado eficiente nos estudos realizados por Medeiros e Torezan (2013) e Graciano-Silva et al (2018) para Floresta Estacional Semidecidual. Já "espécies exóticas lenhosas" apresentou bons resultados no estudo de Graciano-Silva, Mello e Cardoso-Leite (2018) por se tratar de uma área com matriz urbana, onde a invasão de espécies exóticas é bastante grande. Já os indicadores $C$ (cobertura de gramíneas) e $\mathrm{K}$ (espécies tardias do sub-bosque) estiverem presentes na maioria das parcelas, portando sendo considerados indicadores de eficiência regular (Figura $2 \mathrm{C}, \mathrm{K}$ ). Os indicadores B, E, F, G, H e I (Figura 2) variaram muito entre as parcelas, ou seja, conseguiram evidenciar as variações entre as mesmas, sendo considerados indicadores eficientes para análise de integridade em Floresta Ombrófila Densa.

Comparando os resultados das parcelas (Tabela 3) e da área em geral da RPPN com os resultados da área de referência (PECB) pode-se observar que, em geral, para a maioria dos indicadores, as notas do PECB foram maiores e, portanto, a integridade da área foi considerada boa, e da RPPN foi considerada regular. Esse resultado era esperado, uma vez que a área do PECB é muito maior, possui um entorno florestal e há muito tempo vem sendo mantida como Unidade de Conservação.

Para os indicadores $A, C, D$ as notas não foram muito diferentes entre as parcelas da RPPN e do PECB, ou seja, tanto na RPPN quanto no PECB a incidência de serapilheira, e a ausência ou raridade de gramíneas e espécies exóticas também foi comum entre as duas áreas. Isso indica que para a análise da integridade da área de estudo, tendo como referência a área do PECB, esses indicadores não foram eficientes.

As notas atribuídas ao indicador "fetos arborescentes" variou bastante tanto na RPPN quanto no PECB. A ocorrência dessas espécies parece estar mais associada com a variação de umidade dentro das áreas, do que com a integridade do ecossistema, pois as mesmas ocorrerem em áreas úmidas e sombreadas (CASTELLO; COELHO; CARDOSO-LEITE, 2017).

Os indicadores que mostraram as diferenças entre a RPPN e o PECB foram principalmente "Euterpe edulis", "epífitas", "clareiras" e "cipós e lianas". Os dois primeiros (Euterpe edulis e epífitas) registraram altos valores para o PECB e baixos para a RPPN, ou seja, na RPPN encontraram-se poucos indivíduos dessas espécies. E os dois seguintes (cipós/lianas e clareiras) apresentaram altos valores na RPPN e baixos no PECB. Dessa forma, pode-se dizer que a vegetação de FOD do PECB encontra-se muito mais íntegra do que a da RPPN de Trápaga.

Toda a região do Vale do Ribeira e Alto Paranapanema sofre pressão pela extração ilegal (INSTITUTO FLORESTAL, 2008) do palmito juçara (Euterpe edulis). Muito provavelmente, devido à falta de fiscalização anteriormente à criação da RPPN Trápaga, a área sofreu com essa extração ilegal, acarretando em baixa densidade dessa árvore nas parcelas amostradas. A área sofreu cortes seletivos de árvores de dossel ("árvores de madeira de lei”) há mais de três décadas (SCARASCIA, 2016). Esses cortes, muito provavelmente, influenciaram em toda a análise das variáveis de integridade biótica, incluindo a ocorrências dessas espécies adultas. Assim, em poucas parcelas foi registrada a ocorrência das espécies selecionadas (indicador J).

Apesar da Floresta Ombrófila Densa apresentar boas condições para a presença de epífitas, pois são florestas úmidas, a RPPN Trápaga apresentou baixa ocorrência das mesmas. A pontuação registrada pode estar associada com o efeito de borda (ARMELIN; MANTOVANI, 2001) originado pelo uso agrícola do solo no entorno na área da RPPN, pois antes da implantação dos cultivos agrícolas a floresta foi fragmentada dentro da Fazenda Lageado (Figura 1) criando uma borda entre a área de mata nativa (RPPN) e o restante da Fazenda (áreas agrícolas). Sabe-se que o efeito de borda provoca diminuição da umidade relativa do ar e aumento da ocorrência de ventos (MURCIA, 1995) e isso deve estar afetando negativamente a presença de epífitas.

A ausência de continuidade (ou fechamento) do dossel é um fator que está associado com a queda de árvores, ou mesmo presença de árvores mortas em pé, indicando perturbações locais, 
ou seja, a presença de clareiras na RPPN está indicando que existem perturbações na área, assim como a predominância de cipós finos e emaranhados também indicam perturbações (FONSECA; OLIVEIRA, 1998). Essas perturbações podem estar vinculadas ao histórico de uso da área, e aos cortes seletivos de madeira realizados há décadas (INSTITUTO FLORESTAL, 2008).

Apesar da RPPN Trápaga demonstrou um grande número de parcelas com integridade regular, a área apresentou poucas espécies exótica lenhosas, o que indica que a área não está sofrendo invasão biológica por espécies lenhosas. Além disso, apresentou também poucas gramíneas exóticas e um sub-bosque com alta densidade de espécies tardias. Segundo Castello, Coelho e Cardoso-Leite (2017), a presença de espécies tardias no sub-bosque pode ser indicativo de uma de regeneração natural da área.

Medeiros e Torezan (2013) estudaram áreas de FES com tamanho entre 2 a 850 hectares, e registraram IIB variando entre 24 a 50, sendo que algumas das áreas são legalmente protegidas. Já Graciano Silva, Melo e Cardoso-Leite (2018) adaptaram o método para "Florestas Urbanas" e estudaram áreas entre 0,32 e 31 hectares, registrando resultados de IIB entre 22,3 a 35,3. Embora esses estudos tenham sido elaborados para FES, com base nesses números, pode-se dizer que o resultado obtido no presente estudo está coerente, pois o IIB para RPPN Trápaga foi maior do que aquele registrado para todas as florestas urbanas estudadas por Graciano-Silva, Mello e Cardoso-Leite (2018) e comparável com a maioria das áreas estudadas por Medeiros e Torezan (2013).

\section{Conclusões}

O IIB mostrou-se eficiente para análise da integridade biótica da FOD, pois conseguiu mostrar a diferença de integridade entre diferentes trechos de Floresta Ombrófila Densa, representados dentro de uma UC de Proteção Integral e uma RPPN.

Os indicadores menos eficientes foram: "cobertura de serapilheira" e "espécies exóticas lenhosas'; e os mais eficientes foram: "Euterpe edulis", "epífitas", "clareiras" e "cipós/lianas".

A área da RPPN apresentou integridade regular, o que era esperado devido ao seu tamanho, entorno agrícola e histórico de perturbações. Os indicadores "Euterpe edulis" "epífitas", "clareiras" e "cipós/lianas" podem ser utilizados para um monitoramento comparativo da área, podendo também ser utilizados no monitoramento de outras Unidades de Conservação, em região de Floresta Ombrófila Densa.

\section{Referências}

ALLEN, C. D. Monitoring environmental impact in the upper sonoran lifestyle: a new tool for rapid ecological assessment. Environmental Management, [s. l.], v. 43, p. 346-356, 2009.

ARMELIN, R. S.; MANTOVANI, W. Definições de clareira natural e suas implicações no estudo da dinâmica sucessional em florestas. Rodriguésia, Rio de Janeiro, v. 52, n. 81, p. 5-15, 2001.

$\mathrm{BIZ}$, S. et al. Invasão de gramíneas exóticas em áreas sob diferentes tecnologias de restauração florestal. In: CONGRESSO DE CIÊNCIA E TECNOLOGIA DA UTFPR,2.; SEMINÁRIO: SISTEMAS DE PRODUÇÃO AGROPECUÁRIA, 6.; SIMPÓSIO DE CIÊNCIAS FLORESTAIS E BIOLÓGICAS, 2., 2012, Curitiba. [Anais]... Curitiba: Universidade Tecnológica do Paraná, 2012.

BOSCOLO, D. et al. Positive Responses of flower visiting bees to landscape heterogeneity depending on functional connectivity levels. Perspectives in Ecology and Conservation, [s. l.], v. 15, p. 18-24, 2017. 
BRASIL. Lei n. 9.985, de 18 de julho de 2000. Sistema Nacional de Unidades de Conservação da Natureza, Brasília. Disponível em: http://www.planalto.gov.br/ccivil_03/leis/19985.htm.

CASTEllo, A. C. D.; COELHO, S.; CARDOSO-LEITE, E. Lianas, tree ferns and understory species: indicators of conservation status in the Brazilian Atlantic Rainforest Remnants, Southeastern Brazil. Brazilian Journal of Biology, São Carlos, v. 77, n. 2, p. 213-226, 2017.

FONSECA, R. C.; OLIVEIRA, R. E. Ecologia de Lianas e o Manejo de Fragmentos Florestais. Série Técnica IPEF, São Paulo. v. 12, n. 32, p. 43-64, 1998.

FRANKLIN, J. F.; SHUGART, H. H.; HARMON, M. E. Tree Death as na Ecological Process. BioScience, Oxford. v. 37, p. 550-556, 1987.

GANDOLFI, S.; JOLY, C. A.; LEITÃO FILHO, H. F. "Gaps of deciduousness": cyclical gaps in tropical forests. Scientia Agricola, São Paulo, v. 66, n. 2, p. 280-284, 2009.

GASPER, A. L.et al. Quantidade de Árvores Mortas em Pé nas Florestas de Santa Catarina. In: CONGRESSO NACIONAL DE BOTÂNICA, 64., 2013, Belo Horizonte, MG. [Anais]... Belo Horizonte: [s. n.], 2013.

GRACIANO-SILVA, T. Análise e Estabelecimento de Índice de Integridade Biótica para Florestas Urbanas. 2016. Dissertação (Mestrado em Sustentabilidade na Gestão Ambiental) Universidade Federal de São Carlos, Sorocaba, 2016.

GRACIANO-SILVA, T.; MELLO, K.; CARDOSO-LEITE, E. Adaptação e Eficiência de um Índice de Integridade Biótica para análise da sustentabilidade em florestas urbanas. Gaia Scientia, Paraíba, v. 12, n. 2, p. 60-75, 2018.

GREGORINI, R. A. Análise de Áreas para Criação de Unidades de Conservação no Município de Boituva (SP). 2015. Dissertação (Mestrado em Sustentabilidade na Gestão Ambiental) Universidade Federal de São Carlos, Sorocaba, 2015.

HOCKINGS, M.; STOLTON, S.; DUDLEY, N. Evaluating effectiveness: a framework for assessing management of protected areas. Cambridge: IUCN Cardiff University Best Practice Series, 2000.

INSTITUTO FLORESTAL (SP). Plano de Manejo: Parque Estadual Carlos Botelho. São Paulo, 2008.

INTERNATION UNION FOR CONSERVATION OF NATUREZA. Protected areas. [2019]. Disponível em: https://www.iucn.org/theme/protected-areas. Acesso em: nov. 2019.

KOZERA, C.; RODRIGUES, R. R.; DITTRICH, V. A. O. composição florística do sub-bosque de uma floresta ombrófila Densa Montana, Morretes, PR, Brasil. Floresta, Curitiba, v. 39, n. 2, p. 323-334, 2009.

LE SAOUT, S. et al. Protected Areas and Effective Biodiversity Conservation. Science, London, v. 342, n. 6160, p. 803-805, 2013.

MANIA, L. F. Composição florística de comunidades epifíticas vasculares em unidades de conservação no Estado de São Paulo. 2013. Tese (Doutorado em Ciências Biológicas) Universidade Estadual Paulista, São Paulo, 2013.

MEDEIROS, H. R.; TOREZAN, J. M. Evaluating the ecological integrity of Atlantic Forest Remnants by using rapid ecological assessment. Environmental Monitoring and Assessment, Maine, v. 185, p. 4373-4382, 2013.

MITTERMEIER, R. A. et al. Biodiversity hotspots and major tropical wilderness areas: approaches to setting conservation priorities. Conservation Biology, Melbourne, v. 12, n. 3, p. 516-520, 1998.

PAVIOLO, A. et al. A Biodiversity hotspot losing its top predator: the challenge of jaguar 
conservation in the Atlantic Forest of South America. Scientific Reports, [s. l.] n. 6, p. 1-16, 2016. MURCIA C. Edge effects in fragmented forests: implications for conservation. Trends in Ecology \& Evolution, [s. l.], v. 10, n. 2, p. 58-62, feb. 1995. DOI: 10.1016/S0169-5347(00)88977-6.

PIVELLO, V. R.; FREITAS, G. K. A Ameaça das Gramíneas Exóticas à Biodiversidade. O desafio da conservação dos recursos naturais na região. In: O DESAFIO da Conservação dos Recursos Naturais da Região. São Paulo: Secretaria do Meio Ambiente do Estado de São Paulo, 2008. p.234248.

SAYRE, R. et al. Nature in Focus: rapid ecological assessment.1. ed. Washington: Island, 2000.

SCARASCIA, P. O. Licenciamento Ambiental de Turismo Sustentável em Reserva Legal, São Miguel Arcanjo, São Paulo. 2016. Trabalho de Conclusão de Curso (Especialização em Gestão Ambiental e Sustentabilidade) - Universidade Federal de São Carlos, Sorocaba, 2016.

SICHE, R. etal. Índices versus Indicadores: precisões conceituais na discussão da sustentabilidade de países. Ambiente e Sociedade, [s. l.], v. 10, n. 2, p. 137-148, 2007.

SOBREVILLA, C.; BATH, P. Evaluacion Ecologica Rapida: um manual para usuarios de America Latina y el Caribe. Washington, The Nature Conservancy, Virginia, 1992

STEIN, E. D.; FETSCHER, A. E.; CLARK, R. P. Validation of a wetland rapid assessment method: use of EPA's Level 1-2-3. Framework for Method Testing and Refinement. Wetlands, [s. l.] v. 29, n. 2, p. 648-665, 2009.

SUTULA, M. A. et al. A Practical guide for the development of a wetland assessment method: the California experience. Journal of the American Water Resources Association, [s. l.], v. 42, p. 157-175, 2006.

TURCHETTO, F; FORTES, F. O. Aporte e Decomposição de Serapilheira em Floresta Estacional Decidual na região do Alto Uruguai, RS. Pesquisa Florestal Brasileira, Colombo, v. 34, n. 80, p. 391-397, 2014. 